\title{
BI-DIRECTIONAL FLEXIBLE PNEUMATIC ACTUATOR
}

\author{
Carlo FERRARESI, Walter FRANCO and Giuseppe QUAGLIA \\ Department of Mechanics, Politecnico di Torino \\ Corso Duca degli Abruzzi 2410129 Torino, Italia \\ (E-mail ferraresi@polito.it)
}

\begin{abstract}
This paper features the development of a double-effect deformable actuator, conceived as the synthesis of a fluid muscle actuator and a pneumatic spring. The actuator is therefore able to exert both compression and tension forces. The paper describes the scheme of the actuator and the design solution adopted. Subsequently the results of experimental tests for the static characterisation are presented. The results show the compression and tension force of the actuator as a function of supply pressure and stroke. These experimental characteristics are evaluated, discussed and compared with the theoretical results provided by an analytical model. Finally, some possibilities for application and further development of the actuator are proposed.
\end{abstract}

\section{KEY WORDS}

Pneumatics, Flexible Actuator, Pneumatic Spring, Pneumatic Muscle

\section{NOMENCLATURE}

$\begin{array}{ll}\text { A } & \text { end plates area }=\pi \cdot \mathrm{r}^{2}, \mathrm{~m}^{2} \\ \mathrm{a}, \mathrm{b} & \text { quadratic approximations constants } \\ \mathrm{F} & \text { actuator force, } \mathrm{N} \\ \mathrm{K}=\mathrm{L}_{\mathrm{F}} / \mathrm{r} & \text { slimness ratio } \\ \mathrm{L} & \text { actuator length, } \mathrm{m} \\ \mathrm{L}_{\mathrm{F}} & \text { membrane fibres length, } \mathrm{m} \\ \mathrm{p} & \text { internal relative pressure, } \mathrm{Pa} \\ \mathrm{r} & \text { external plates radius, } \mathrm{m} \\ \mathrm{R} & \text { curvature radius, } \mathrm{m} \\ \mathrm{t} & \text { membrane thickness, } \mathrm{m} \\ \tau & \text { membrane axial stress, } \mathrm{N} / \mathrm{m}^{2} \\ \phi & \text { curvature arc half-angle, } \mathrm{m}\end{array}$

\section{INTRODUCTION}

Pneumatic muscles are deformable fluid actuators able to contract and to exert a tension force when supplied with a given pressure. Among the different typologies of actuators we cite the McKibben muscle, conceived in the 1950s, and the straight fibres muscle [1], [2].

Pneumatic springs are deformable fluid actuators as well, able to bear a compression force, or to exert a pushing force. The first examples of pneumatic spring came out at the end of the $19^{\text {th }}$ century. Some design and modelling techniques are reported in [3], [4].

Both typologies of actuators present some peculiar advantages, such as: low mass, high force/mass ratio, lack of sliding parts, no need of lubrication, the possibility of working with cheap and non-polluting fluids (air, water), the possibility of moving structures kinematically non-defined, the possibility of working in extreme environments (absence of atmosphere, heavy temperature gradients). Therefore such actuators may be suitably employed to actuate structures for robotic, biomechanical and aerospace applications.

On the contrary, muscle actuators and pneumatic springs have the notable disadvantage of exerting only mono-directional forces, therefore they cannot be used in applications that require a double-effect actuation, 
unless installing two actuators operating with the principle of antagonistic muscles.

Starting from these considerations, we conceived the idea to develop an actuator able to exert both tension and compression forces. Such idea led to the draft of a patent and to the realisation of a prototype, whose characteristics are described in this paper.

The article features the actuator's working principle, proposes an analytical model and shows the prototype realised. Then the static characteristics recorded experimentally are reported. The model's validity is discussed. Finally some possibilities of evolution of the actuator are proposed.

\section{WORKING PRINCIPLE AND FIELDS OF APPLICATION}

The actuator is made up of two cylindrical, coaxial deformable membranes, connected to two end plates (heads) in such a way as to generate two coaxial chambers: an external annular chamber (A) and an internal chamber $(\mathrm{B})$, as represented in figure 1 .

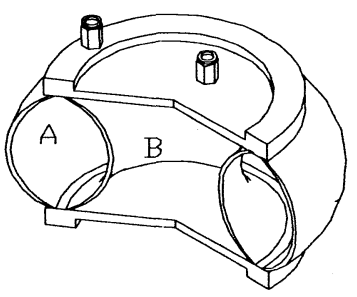

Figure 1 The scheme of the actuator (section)

The membranes present high deformability in the circumferential direction and very low deformability in the longitudinal direction. Such characteristic has been obtained by using a rubber membrane which has been stiffened in one direction by inserting proper fibres in the elastomeric matrix.

The two heads allow to supply the two coaxial chambers, guarantee the sealing and allow the connection of the actuator to the structure that has to be moved.

If one supplies the external chamber with pressurised fluid, the membranes expand only in the circumferential direction and not in the longitudinal one, so causing the approach of the heads, that is exerting a tension force. The actuator is therefore in the tension configuration.

On the contrary, by supplying at the same time both the external and internal chamber, the thrust of the pressure on the heads causes the extension of the actuator, or allows to exert a thrust on the structure connected. In this case the actuator is in the compression configuration.

This kind of actuator presents many advantages. First of all it works only by means of a deformation of the structure and therefore without sliding between different parts: this implies that no friction or wear is present in normal working condition; in addition the risk of fluid leakage is almost null. The actuator is characterised by an intrinsic deformability, which allows it to be employed in bi-directional actuation of structures kinematically non defined. It is able to operate with different kind of working fluids, both liquid or gas. When supplied with compressible fluid, it presents an intrinsic compliance, that allows its application in the field of vehicular suspensions and of the active insulation of vibrations. It can work within strong electromagnetic fields. Because of the easiness of sterilisation, it can be used in biomedical environment, for the actuation of structures in clean rooms.

\section{STATIC MATHEMATICAL MODEL}

The analytical static model must be able to express the force exerted by the actuator as a function of its height, given internal pressure and slimness ratio $\mathrm{K}$.

The model is based on the following simplifying hypotheses:

- the membrane has a circular contour with curvature radius $\mathrm{R}$, in longitudinal sections;

- the membrane axial stress can be calculated transforming the closed axial symmetric contour in an open surface with the same longitudinal contour, as shown in figure 2 ;

- the axial stiffness of membrane fibres is infinite, so that fibres can be considered non-extendable;

- the fibres circumferential stiffness is negligible.
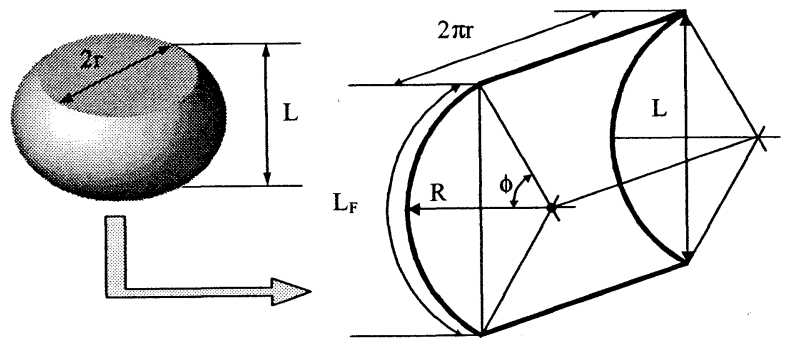

Figure 2 Simplified geometry obtained from axialsymmetric convolution

First the analytical model for compression configuration is developed, then the model is extended to the tension configuration.

Longitudinal stress and geometrical aspects of the membrane can be obtained from figure 2 , where the geometrical simplification is applied.

Figure 3 (left) shows the forces acting on the simplified membrane contour having thickness $t$, leading to the following equilibrium equation:

$$
p \cdot L \cdot 2 \pi \cdot r=2 \cdot \tau \cdot t \cdot 2 \pi \cdot r \cdot \sin \phi
$$



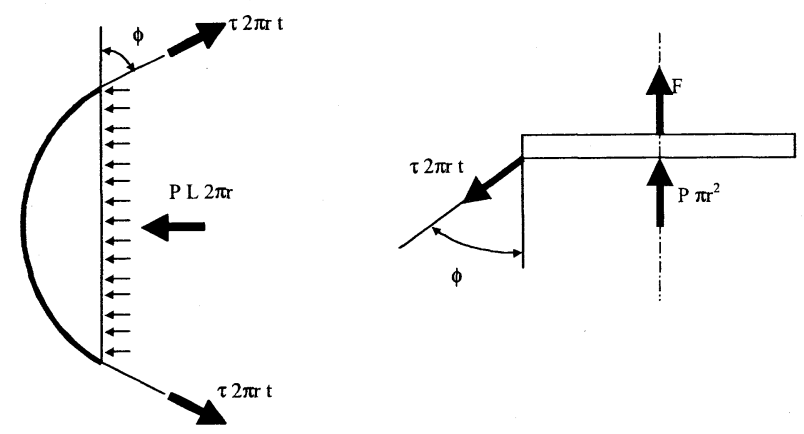

Figure 3 Equilibrium schemes of membrane and upper plate

Figure 3 (right) shows the forces acting on the actuator's upper plate. Its axial equilibrium equation puts out the relations between external force $F$, internal pressure $p$ and membrane configuration, as a function of angle $\phi$ :

$$
F=\tau \cdot t \cdot 2 \pi r \cdot \cos \phi-p \cdot A
$$

Deriving $\tau$ from (1) and substituting in (2), the force $F$ is obtained:

$$
F=\frac{L \cdot p}{\sin \phi} \cdot \pi r \cdot \cos \phi-p \cdot A
$$

Equation (3) can be simplified finding the relation between auxiliary angle $\phi$ and length $\mathrm{L}$. Since the fibres are considered longitudinally non-extendable, this leads to:

$$
R \cdot 2 \phi=L_{F}
$$

A section of actuator membrane is supposed to be a circular arc, with radius $R$ (figure 2 ). This arc subtends an angle, which value is $2 \phi$ :

$$
R \cdot \sin \phi=L / 2
$$

using (4) and (5), it can be derived that:

$$
y(\phi)=\frac{L}{L_{F}}=\frac{\sin \phi}{\phi}
$$

Using (3) and (6), and introducing the slimness ratio parameter $\mathrm{K}=\mathrm{L}_{\mathrm{F}} / \mathrm{r}$, the following non-linear equation system is obtained:

$$
\left\{\begin{array}{c}
\frac{F}{p \cdot A}=K \frac{\cos \phi}{\phi}-1 \\
\frac{L}{L_{F}}=\frac{\sin \phi}{\phi}
\end{array}\right.
$$

This equation system expresses the non-dimensional actuator force $(\mathrm{F} /(\mathrm{p} \cdot \mathrm{A}))$ versus the non-dimensional actuator length $\left(\mathrm{L} / \mathrm{L}_{\mathrm{F}}\right)$.

In (7) the auxiliary angle $\phi$ is still used to link the two equations. It is not possible to find one single equation to express the non-dimensional force versus the nondimensional length, because the function $\mathrm{y}(\phi)$ cannot be analytically inverted. The function $y(\phi)$ can be approximated with a function $\mathrm{y}_{1}$, that can be reversed. A quadratic expression is chosen:

$$
y_{1}=a \phi^{2}+b
$$

with $a=\frac{8-4 \pi}{\pi^{3}}$ and $\mathrm{b}=1$, resulting from imposing $y(0)=1$ and $y(\pi / 2)=2 / \pi$.

The inverse relation expresses $\phi$ versus the actuator length $\mathrm{L}$ :

$$
\phi_{1}=\sqrt{\frac{L / L_{F}-1}{a}}
$$

Inserting expression (9) into (7), the non-dimensional force as a function of non-dimensional actuator length is directly obtained:

$$
\frac{F}{p \cdot A} \cong K \frac{\cos \left(\sqrt{\frac{\frac{L}{L_{F}}-1}{a}}\right)}{\sqrt{\frac{\frac{L}{L_{F}}-1}{a}}}-1
$$

The equation (10) for the compression configuration evidences that the static behaviour is directly influenced by the slimness ratio $\mathrm{K}$.

In a similar way the equation describing the behaviour in the tension configuration can be derived:

$$
\frac{F}{p \cdot A} \cong 2 K \frac{\cos \left(\sqrt{\frac{\frac{L}{L_{F}}-1}{a}}\right)}{\sqrt{\frac{\frac{L}{L_{F}}-1}{a}}}
$$

The equations (10) and (11) are plotted in figure 4. This graph may be a valid help to dimension the actuator for a specific application. In fact, slimmer actuators $(\mathrm{K}>1)$ 
are more suitable in cases that require higher performance in tension mode, while dumpy actuators may be preferred when working prevalently in compression condition.

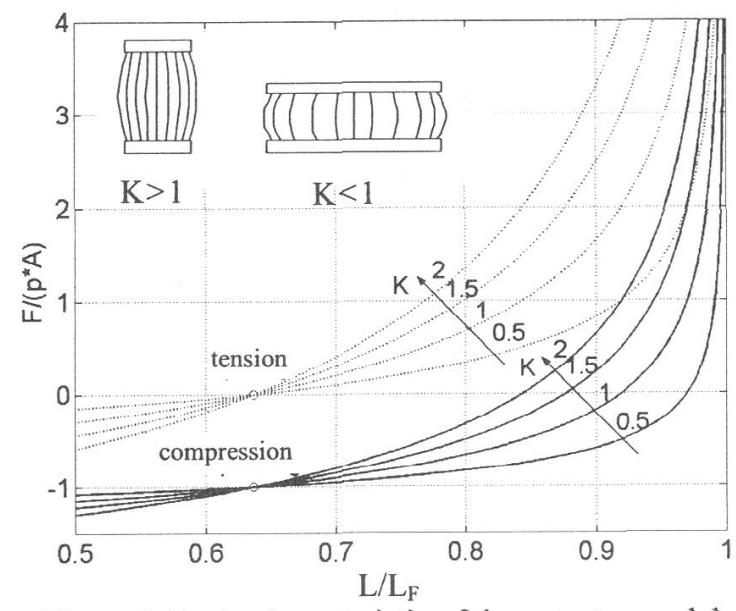

Figure 4 Static characteristic of the actuator model

\section{THE PROTOTYPE}

The prototype realised is shown in the figure 5 .

The design solution adopted is reported in figure 6 .

The main technical difficulties concerned the realisation of the membranes, their connection to the heads and the supply of the external chamber.

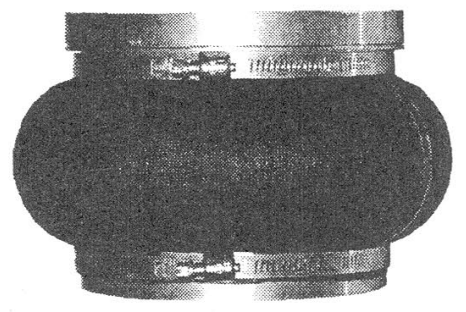

Figure 5 The prototype

In the rest condition, the membranes present a cylindrical tubular shape. They have been built starting from a rectangular sheet of S.B.R. vulcanized rubber, $1.5 \mathrm{~mm}$ thick, including a net of polyester fibres oriented along only one direction. Two sides of the sheet have then been glued together, so as to obtain the cylindrical shape, by means of a cyano-acrylic adhesive, in such a way that the fibres were parallel to the longitudinal axis of the tube.

The connection of the membranes to the heads has been realised with different techniques for the external and the internal membrane.

The internal membrane has been mounted on a disc having tapered edge. The membrane is tightened between the disc and an external ring by means of a flange and proper screws.

The external membrane has been fixed to the same ring by means of a hose clamp.

These two different kinds of tightening showed different results: the system with cone and counter-cone turned out to be the most effective, while the hose clamp for the external membrane is not suitable for pressures higher than 1 bar.

Finally, the supply of the external chamber has been realised by means of a threaded port and an annular groove that distributes the air among sixteen holes made on the ring.

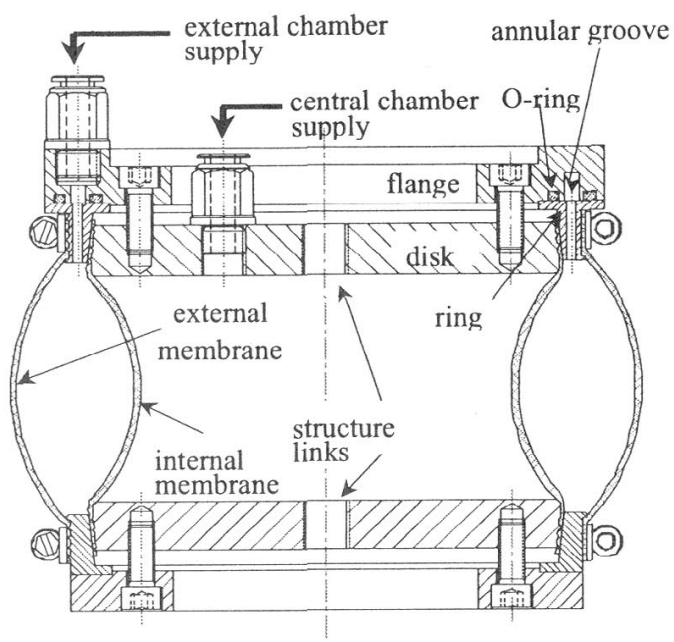

Figure 6 Sectional view of the actuator

\section{THE EXPERIMENTAL TESTS}

The static characterisation of the actuator has been carried out by means of a proper test bench. Here a hydraulic servo-actuator imposes the relative position of the prototype heads, while pressure-proportional pneumatic valves maintain the internal pressures equal to constant preset values. The position of the heads is read by means of a LVDT (F.S. $200 \mathrm{~mm}$, linearity $1 \%$ F.S.), while the load applied to the actuator is measured by means of a load cell (F.S. $5000 \mathrm{~N}$, linearity $0.05 \%$ F.S.).

The tests have been carried out by measuring the force exerted by the actuator at different contraction values, imposed by the hydraulic servo-system, and for different supply pressures imposed by the proportional valves.

The actuator has been tested both in the tension configuration (only the external chamber supplied) and in the compression configuration (internal and external chamber supplied at the same time).

Besides two different actuators, having respectively slimness ratio of 1.25 and 1.85 , have been tested. The 
two actuators have been realised mounting on the same heads membranes of different height. The geometrical data of the two actuators are reported in table 1, where the apexes $e$ and $i$ indicate the characteristics concerning respectively the external and internal membrane.

Table 1 Geometrical data of the actuators

\begin{tabular}{|c|c|c|c|c|c|c|}
\hline prototype & $\begin{array}{c}\text { mass } \\
{[\mathrm{kg}]}\end{array}$ & $\begin{array}{c}\mathbf{L}_{\mathbf{F}}{ }^{\mathbf{e}} \\
{[\mathrm{mm}]}\end{array}$ & $\begin{array}{c}\mathbf{L}_{\mathbf{F}}^{\mathbf{i}} \\
{[\mathrm{mm}]}\end{array}$ & $\begin{array}{c}\mathbf{r}^{\mathbf{e}} \\
{[\mathrm{mm}]}\end{array}$ & $\begin{array}{c}\mathbf{r}^{\mathbf{i}} \\
{[\mathrm{mm}]}\end{array}$ & $\mathbf{K}$ \\
\hline$\# 1$ & 3.988 & 74 & 63 & 56.5 & 50.7 & 1.25 \\
\hline$\# 2$ & 4.016 & 109 & 90 & 56.5 & 50.7 & 1.85 \\
\hline
\end{tabular}

The figures 7 and 8 show the static characteristic of the actuator force versus contraction, for the actuator with slimness ratio of 1.25 , for the tension and compression configuration respectively.

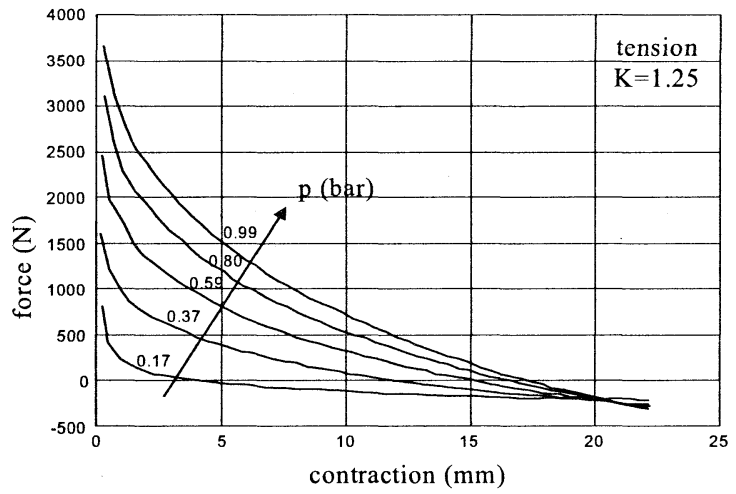

Figure 7 Tension static characteristic

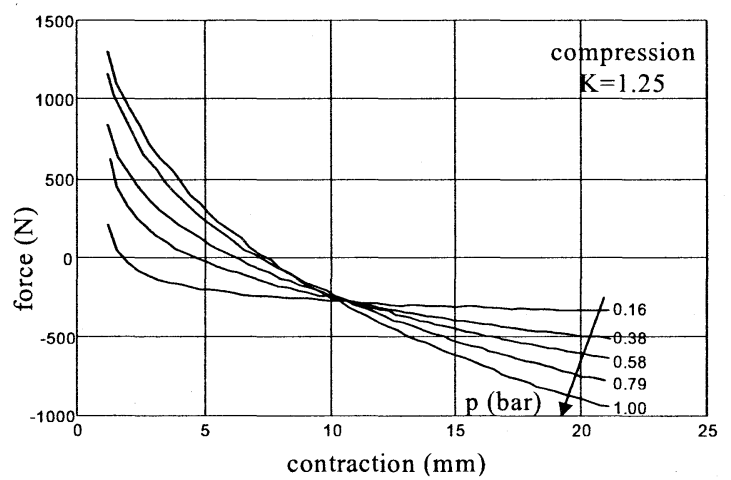

Figure 8 Compression static characteristic

A positive value indicates a tension force. Null contraction corresponds to the condition of non supplied actuator, with no external force $\left(\mathrm{L}=\mathrm{L}_{\mathrm{F}}\right)$.

The analysis of the experimental results highlights that the actuator, when supplied at constant pressure, exerts, both in tension and compression condition, a force which decreases when the contraction increases.

This means that, in tension condition, an increase of the contraction determines a reduction of the absolute value of the force. On the contrary, in compression state, when the contraction increases the absolute value of the force increases as well.

Besides for both working conditions a given contraction value exists, where the force exerted by the actuator is practically independent from the supply pressure.

It is important to notice that, in both configurations, when the actuator contraction varies, an inversion of the force sign may be observed. The actual field of utilisation is therefore limited with respect to what indicated in figures 7 and 8.

In tension configuration (figure 7), for actuator contraction corresponding to a positive force (so actually a tension force), the value of the force increases as the supply pressure increases.

In compression configuration (figure 8), on the contrary, for actuator contraction corresponding to a negative force (so actually a compression force), in a first range of contraction the thrust force decreases as the supply pressure increases, then, for contraction greater than 10 $\mathrm{mm}$, the force increases as the pressure increases.

The figure 9 shows the effect of the slimness ratio $\mathrm{K}$.

One can notice that, at same supply pressure, an actuator with greater $\mathrm{K}$ ratio is able to exert, in tension configuration, greater tension forces, while an actuator having lower $\mathrm{K}$ ratio can exert, in compression configuration, greater thrust forces.

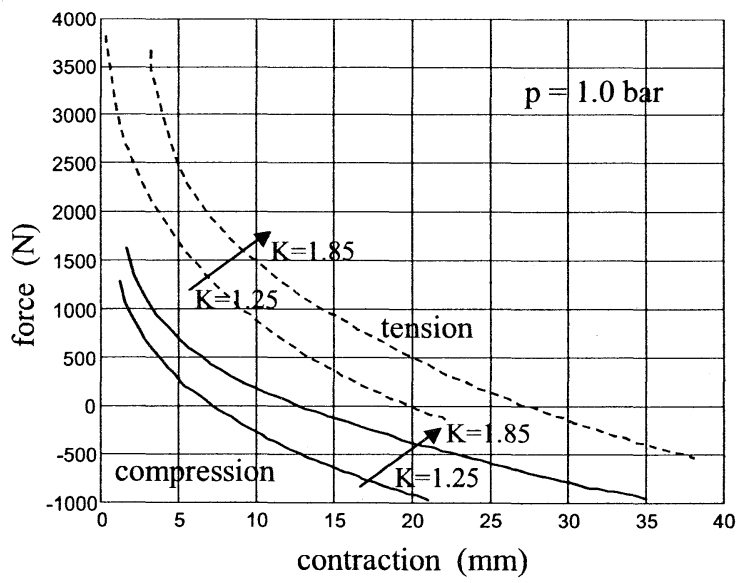

Figure 9 Compression and tension static characteristic

\section{MODEL VALIDATION}

The figure 10 shows a comparison between the experimental and numerical characteristics in the case of actuator with slimness ratio 1.25. 
The graph reports the nondimensional tension force as a function of the nondimensional actuator length.

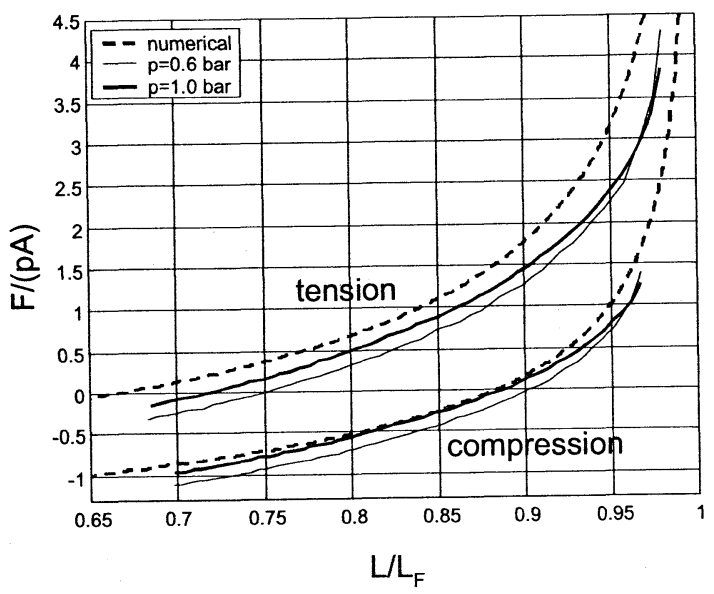

Figure 10 Comparison between numerical and experimental characteristics

The model shows a good agreement with the experiment, although the nondimensional force actually depends also on the supply pressure.

The model is based on ideal conditions, therefore it produces always an optimistic prevision of the actuator force. Besides, it is worth noting that the discordance between experimental and numerical results is partially due to the difficulty of determining the null contraction position during the tests, because of the intrinsic compliance of the actuator.

\section{FUTURE DEVELOPMENTS}

The actuator presents interesting possibilities of development, concerning both the realisation of different variants, and the methodologies of use.

A first consideration must be done as concerns the proportions of the actuator: by varying properly the ratio between length and diameter it is possible to realise actuators with different characteristics, adaptable to the specific application.

Then the heads may be arranged so as to link two or more actuators in series, producing an actuator with a stroke greater than that of a single module (figure 11 (a)).

The same result can be obtained by building an actuator with very high slimness and applying proper internal and external rings to restrain the circumferential deformation of the membranes (figure 11 (b)).

Finally, techniques for an "energy saving" use of the actuator may be adopted. A possibility consists in placing within the central chamber a body made of inert material, with the aim to reduce the mass of the working fluid (figure 12).

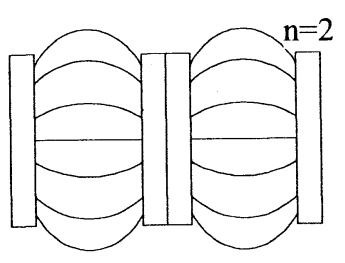

(a)

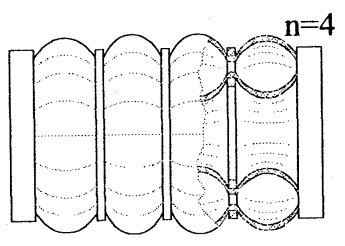

(b)
Figure 11 actuators in series:

(a) modular actuators; (b) multiple actuator

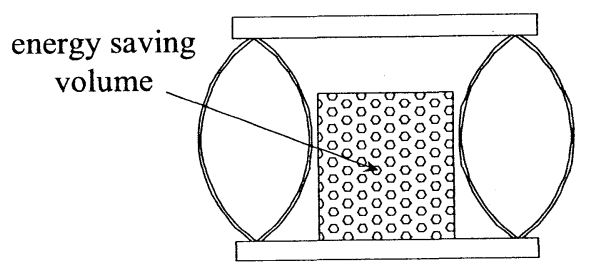

Figure 12: energy saving actuator, with filling inert body

\section{ACKNOWLEDGEMENTS}

The authors wish to thank Dr. Eng. Paolo Gatti for the co-operation in the prototype development and in the experimental tests, and the New Rubbertex company (Italy) for the help in seeking and providing the elastomeric material of the membranes.

This work has been supported by the Italian Space Agency (ASI) in the frame of the project "Deformable fluid actuators for space applications".

\section{REFERENCES}

1. Ferraresi, C., Franco, W., Manuello Bertetto, A., Straight fibres pneumatic muscle: an actuator with high traction force, The Sixth International Conference on Fluid Power, SICFP'99, May 26-28 1999, pp. 787-798

2. Ferraresi, C., Franco, W., Manuello Bertetto, A., Flexible Pneumatic Actuators: a Comparison Between the McKibben and the Straight Fibres Muscles Journal of Robotics and Mechatronics, Fuji Technology Press, Tokyo, Japan, Vol. 13, n.1, 2001, pp.56-63

3. Quaglia G., Sorli M, "Analisi di Sospensioni Veicolari Pneumatiche", Oleodinamica e Pneumatica, ed. Tecniche Nuove, $n^{\circ} 9$ ottobre 2000, pp 76-83

4. Quaglia G., Sorli M, "Air Suspension Dimensionless Analysis and Design Procedure", Vehicle System Dynamics, Vol. 35, No 6, 2001, pp. $443-475$ 